\title{
ON THE DYNAMICS OF A VACCINATION MODEL WITH MULTIPLE TRANSMISSION WAYS
}

\author{
SHU LIAO, WEIMING YANG \\ School of Mathematics and Statistics \\ Chongqing Technology and Business University, Chongqing, 400067, China \\ e-mail: shuyang2011@yahoo.com
}

\begin{abstract}
In this paper, we present a vaccination model with multiple transmission ways and derive the control reproduction number. The stability analysis of both the disease-free and endemic equilibria is carried out, and bifurcation theory is applied to explore a variety of dynamics of this model. In addition, we present numerical simulations to verify the model predictions. Mathematical results suggest that vaccination is helpful for disease control by decreasing the control reproduction number below unity.
\end{abstract}

Keywords: vaccination model, stability, equilibrium.

\section{Introduction}

Waterborne diseases, the world's leading killer, are infectious diseases caused by pathogenic microorganisms that are transmitted in contaminated water. According to the World Health Organization (WHO) reports, 3-5 million people are affected by water-related diseases every year. Cholera is an acute intestinal infection caused by bacterium Vibrio cholerae; its transmission is different from other diseases since it involves multiple transmission pathways and occurs primarily by drinking contaminated water or eating food that has been contaminated by the feces of an infected person, including one with no apparent symptoms. Currently, cholera is endemic in over 50 countries, including India (2007), Congo (2008), Iraq (2008), Zimbabwe (2008-2009), Vietnam (2009), Kenya (2010), Nigeria (2010), and most recently Haiti (2010-2011) (WHO).

Recently, the use of compartmental models has proved to be a powerful tool for exploring complex transmission dynamics. There have been published many mathematical models focused on modeling and simulating the nature of the cholera dynamics. Capasso and Paveri-Fontana (1979) framed a simple cholera model to study the 1973 cholera epidemic in the European Mediterranean region. Codeço (2001) first proposed an SIRB epidemic model by adding an environmental component into the regular SIR model ( $B$ is the $V$. cholerae concentration in the water environment).
The extension of Codeço's model by Hartley et al. (2006) accounted for a hyperinfectious state of the pathogen, representing the hyperinfectious state of the pathogen, the explosive infectivity of freshly shed $V$. cholerae, based on the laboratory results. Recently, Liao and Wang (2011) conducted a dynamical analysis of Hartley's model. They studied the stability of both the disease-free and endemic equilibria so as to explore the complex epidemic and endemic dynamics of the disease. Also, Mukandavire et al. (2011) considered both human-to-human and environment-to-human transmission pathways and simplified Hartley's model to study the 2008-2009 cholera outbreak in Zimbabwe. Moreover, Tien and Earn (2010) proposed a water-borne disease model with multiple transmission pathways, and identified how these transmission routes influence disease dynamics.

Although waterborne diseases are severe infections, due to vaccination, advanced water treatments and sanitation practices, which shorten the course of the disease and reduce the severity of the symptoms, these terrible diseases are no longer major health threats in most countries. Vaccination of susceptible, as one of the most effective controlling methods for many diseases (measles, pertussis, rubella, hepatitis $\mathrm{B}$, mumps, influenza, etc.) has been included in mathematical compartmental models and published recently. Many studies have shown that vaccination strategies are very important to control or eradicate diseases (Dietz et al., 1985; Kribs-Zaleta and 
Velasco-Hernandez, 2000; Kribs-Zaleta and Martcherab, 2002; Brauer, 2004; Samsuzzoha et al., 2012; Szymanska, 2013). Dietz and Schenzle (1985) pointed out that realistic epidemic models need to consider vaccination programmes. Kribs-Zaleta and Velasco-Hernandez (2000) explored disease control via a vaccination program in which the vaccine provides complete protection against low-level infection and partial protection against infection by acute infectives. In the work of Kribs-Zaleta and Martcherab (2002), a simple two-dimensional SIS model with vaccination was exhibited and showed a vaccination campaign meant to reduce a disease's reproduction number below which one may fail to control the disease. Brauer (2004) studied the backward bifurcation in simple vaccination models and gave conditions for the existence of multiple endemic equilibria and backward bifurcations. Samsuzzoha et al. (2012) considered an SVEIRS model to explore the impact of vaccination as well as diffusion on the transmission dynamics of influenza. In addition, there are many other epidemic models including different kinds of vaccination (Yildirim and Cherramt, 2009; Song et al., 2009; Yu et al., 2010), such as constant or impulsive.

The aim of this paper is to extend and analyze the waterborne disease model proposed by Tien and Earn (2010) with the inclusion of a vaccinated class, $V$, and three important factors: vaccination rate $\phi$, vaccine efficacy rate $\sigma$ and waning rate $\theta$. The remainder of the paper is organized as follows. In Section 2 we formulate the vaccination model, and the derivation of the control reproductive number $R_{C}$ is included. In Section 3 , we analyze the vaccination model, study the stability of both the disease-free and endemic equilibria. Section 4 is devoted to the numerical study of the model, which confirms our theoretical results. Finally, we close the paper by a discussion in Section 5

\section{ODE model with vaccination}

2.1. ODE model with vaccination and the control reproduction number. Tien and Earn (2010) extended the classical SIR framework by adding a compartment $(W)$ that tracked pathogen concentration in the water. Susceptible individuals are infected with multiple transmission pathways: contact with infected individuals or contact with contaminated water. The corresponding model consists of the following Ordinary Differential Equations (ODEs):

$$
\begin{aligned}
& \frac{\mathrm{d} S}{\mathrm{~d} t}=\mu N-\beta_{W} W S-\beta_{I} S I-\mu S, \\
& \frac{\mathrm{d} I}{\mathrm{~d} t}=\beta_{W} W S+\beta_{I} S I-(\gamma+\mu) I,
\end{aligned}
$$

$$
\begin{aligned}
\frac{\mathrm{d} R}{\mathrm{~d} t} & =\gamma I-\mu R, \\
\frac{\mathrm{d} W}{\mathrm{~d} t} & =\alpha I-\xi W,
\end{aligned}
$$

where $N$ is the total population size divided into three groups: $S, I$ and $R$, denoting the susceptible, the infected and the recovered numbers, respectively. The population size $N$ remains constant, and $S+I+R=N$. Here the parameters $\beta_{W}$ and $\beta_{I}$ represent the transmission rate for environment-to-human and human-to-human, respectively. Here $\mu$ is the natural human birth/death rate, $\alpha$ is the shedding rate, $\xi$ is the bacterial death rate, and $\gamma$ is the recovery rate.

The basic reproduction number $R_{0}$, which represents the average number of secondary infections that occur when one infective is introduced into a completely susceptible host population (Hethcote, 2000; Van den Driessche and Watrauugh, 2002; Vynnycky et al., 2007; Tien and Earn, 2010), is calculated as (Tien and Earn, 2010)

$$
R_{0}=\frac{N\left(\beta_{W} \alpha+\beta_{I} \xi\right)}{\xi(\gamma+\mu)} .
$$

Based on the above model, we formulate a vaccination model and add a new group $V$ that has begun the vaccination process. Because of the new born and those who have loss of immunity due to earlier infection and vaccination with constant rate $\phi$, the number of susceptible individuals is increased. On the other hand, this number is reduced by vaccination, infection and natural death. Moreover, the vaccinated individuals are increased, since the susceptible population is vaccinated and moved to class $V$, the vaccine has the effect of reducing the infection with constant rate $\sigma$, we make the realistic assumption that the vaccine is not totally effective, $0 \leq \sigma \leq 1,(1-\sigma)$ is the vaccine efficacy. Thus, $\sigma=0$ means that the vaccine is completely effective, $\sigma=1$ means that the vaccine has no effect. The number of vaccinated individuals is decreased by natural death, while the infection and waning of vaccine induced immunity at the rate $\theta$. The vaccination model is constructed as

$$
\begin{aligned}
\frac{\mathrm{d} S}{\mathrm{~d} t} & =\mu N-\beta_{W} W S-\beta_{I} S I-(\mu+\phi) S+\theta V, \\
\frac{\mathrm{d} V}{\mathrm{~d} t} & =\phi S-\sigma \beta_{I} V I-(\mu+\theta) V, \\
\frac{\mathrm{d} I}{\mathrm{~d} t} & =\beta_{W} W S+\beta_{I} S I-(\gamma+\mu) I+\sigma \beta_{I} V I, \\
\frac{\mathrm{d} R}{\mathrm{~d} t} & =\gamma I-\mu R, \\
\frac{\mathrm{d} W}{\mathrm{~d} t} & =\alpha I-\xi W,
\end{aligned}
$$

where $S, V, I$ and $R$ denote the susceptible, the vaccinated, the infected, and the recovered numbers, 
respectively. The total population size is $N=S+I+$ $R+V$. Note that in the case of $\sigma=1$, the vaccine is totally useless. Thus the vaccination model reduces to the vaccination free model (1)-(4).

The solution of the model system (6)-10 is biologically feasible for all times. Mathematically speaking, the solution domain

$$
\begin{gathered}
\bar{D}=\{(S, V, I, R) \mid S \geq 0, V \geq 0, I \geq 0, \\
R \geq 0, S+V+I+R=N\},
\end{gathered}
$$

is a positively invariant set in $\mathbb{R}^{4}$.

We can write the ODEs (6)-10) in vector form:

$$
\frac{\mathrm{d} X}{\mathrm{~d} t}=\mathbf{F}(X),
$$

with

$$
X=(S, V, I, R, W)^{T} .
$$

Disease-Free Equilibrium (DFE) points are steady-state solutions where there is no disease. Thus

$$
X_{0}=\left(\frac{N(\mu+\theta)}{\mu+\phi+\theta}, \frac{N \phi}{\mu+\phi+\theta}, 0,0,0\right)^{T} \text {. }
$$

Here, we use the control reproduction number, denoted by $R_{C}$, to describe the average number of secondary cases generated by primary cases under specified controls such as vaccination (Anderson and May, 1990; Hethcote, 2000). It is a useful threshold in the study of a disease for predicting a disease outbreak and for evaluating the control strategies. Based on the work of Van den Driessche and Watmough (2000), the next generation operator approach can be used to define the control reproductive number, where $\mathcal{F}$ denotes the rates of the appearance of new infections in each compartment; $\mathcal{V}=\mathcal{V}^{+}+\mathcal{V}^{-}, \mathcal{V}^{+}$being the vector of individual transfer rates into the particular compartment, and $\mathcal{V}^{-}$ the vector of individual transfer rates out of the particular compartment.

These two vectors are given by

$$
\begin{gathered}
\mathcal{F}=\left[\begin{array}{c}
\beta_{W} W S+\beta_{I} S I+\sigma \beta_{I} V I \\
0 \\
0 \\
0 \\
0
\end{array}\right] \\
\mathcal{V}=\left[\begin{array}{c}
(\gamma+\mu) I \\
\xi W-\alpha I \\
\beta_{W} W S+\beta_{I} S I+(\mu+\phi) S-\theta V-\mu N \\
\sigma \beta_{I} V I+(\mu+\theta) V-\phi S \\
\mu R-\gamma I
\end{array}\right] .
\end{gathered}
$$

The next generation matrix is defined as $F V^{-1}$, where $F$ and $V$ are both the Jacobian matrices given by

$$
F=\left[\frac{\partial \mathcal{F}_{i}}{\partial X_{j}}\left(X_{0}\right)\right], \quad V=\left[\frac{\partial \mathcal{V}_{i}}{\partial X_{j}}\left(X_{0}\right)\right]
$$

with $1 \leq i, j \leq 2$. After some algebra, it is found that

$$
F=\left[\begin{array}{cc}
\beta_{I} S+\sigma \beta_{I} V & \beta_{W} S \\
0 & 0
\end{array}\right]
$$

and

$$
V=\left[\begin{array}{cc}
\gamma+\mu & 0 \\
-\alpha & \xi
\end{array}\right]
$$

The next generation matrix, $F V^{-1}$, has a nonzero eigenvalue corresponding to the spectral radius. It represents the control reproduction number of the model as

$$
R_{C}=\frac{\beta_{W} \alpha N(\mu+\theta)+\beta_{I} \xi N(\mu+\theta+\sigma \phi)}{\xi(\gamma+\mu)(\mu+\theta+\phi)} .
$$

We consider the vaccination rate $\phi$ as a key control parameter and other parameters as fixed. $R_{C}$ depends on the vaccination policy $\phi$ and it is a decreasing function of $\phi$ since $R_{C}^{\prime}(\phi) \leq 0$. When $\phi=0, R_{C}=R_{0}$, and $R_{C}<$ $R_{0}$ for all $\phi>0$ (since $0 \leq \sigma \leq 1$ ). Thus $R_{C}$ reflects how the reproduction number becomes smaller under the application of a vaccination control, and shows how much the vaccination reduces $R_{C}$.

There is a unique $\phi_{C}$, such that $R_{C}\left(\phi_{C}\right)=1$, which can be given as

$$
\phi_{C}=\frac{\left[\beta_{W} \alpha N+\beta_{I} \xi N-\xi(\gamma+\mu)\right](\mu+\theta)}{\xi(\gamma+\mu)-\beta_{I} \xi N \sigma} .
$$

\subsection{Disease-free equilibrium.}

Theorem 1. The disease-free equilibrium of the $O D E$ model (6)-(10) is locally asymptotically stable if $R_{C}<$ 1 , and unstable if $R_{C}>1$.

Proof. Since $R_{C}<1$, we have

$$
N \beta_{I}(\mu+\theta+\phi \sigma)<(\gamma+\mu)(\phi+\mu+\theta) .
$$

The Jacobian of the ODE system (6) - (10) is

$$
\begin{aligned}
& {\left[\begin{array}{cc}
-\beta_{W} W-\beta_{I} I-(\mu+\phi) & \theta \\
\phi & -\sigma \beta_{I} I-(\mu+\theta)
\end{array}\right.} \\
& \beta_{W} W+\beta_{I} I \quad \sigma \beta_{I} I \\
& 0 \\
& 0 \\
& \left.\begin{array}{ccc}
-\beta_{I} S & 0 & -\beta_{W} S \\
-\sigma \beta_{I} V & 0 & 0 \\
\beta_{I} S-(\gamma+\mu)+\sigma \beta_{I} V & 0 & \beta_{W} S \\
\gamma & -\mu & 0 \\
\alpha & 0 & -\xi
\end{array}\right]
\end{aligned}
$$


After substituting the values for the DFE

$$
\begin{aligned}
& S=\frac{N(\mu+\theta)}{\mu+\phi+\theta}, \\
& V=\frac{N \phi}{\mu+\phi+\theta}, \\
& I=R=W=0,
\end{aligned}
$$

the above matrix becomes

$$
J_{B}=\left[\begin{array}{ll}
J_{11} & J_{12} \\
J_{21} & J_{22}
\end{array}\right],
$$

where

$$
\begin{aligned}
& J_{11}=\left[\begin{array}{cc}
(\mu+\phi) & \theta \\
\phi & -(\mu+\theta) \\
0 & 0
\end{array}\right. \\
& -\frac{\beta_{I} N(\mu+\theta)}{\mu+\phi+\theta} \\
& -\frac{\sigma \beta_{I} N \phi}{\mu+\phi+\theta} \\
& \frac{\beta_{I} N(\mu+\theta)}{\mu+\phi+\theta}+\frac{\sigma \beta_{I} N \phi}{\mu+\phi+\theta}-(\gamma+\mu) \\
& J_{12}=\left[\begin{array}{cc}
0 & -\frac{\beta_{W} N(\mu+\theta)}{\mu+\phi+\theta} \\
0 & 0 \\
0 & \frac{\beta_{W} N(\mu+\theta)}{\mu+\phi+\theta}
\end{array}\right] \text {, } \\
& J_{21}=\left[\begin{array}{ccc}
0 & 0 & \gamma \\
0 & 0 & \alpha
\end{array}\right] \text {, } \\
& J_{22}=\left[\begin{array}{cc}
-\mu & 0 \\
0 & -\xi
\end{array}\right] \text {. }
\end{aligned}
$$

The equilibrium is locally asymptotically stable if and only if all the roots of the matrices $J_{11}$ and $J_{22}$ have negative real parts. It is easy to see that the matrix $J_{22}$ has two negative eigenvalues, $(-\mu)$ and $(-\xi)$. After some simple calculation, the two eigenvalues of $J_{11},(-\mu)$ and $(-\phi-\mu-\theta)$ are clearly negative, and according to the inequality (14), the other eigenvalue of $J_{11},-[(\gamma+\mu)(\phi+$ $\left.\mu+\theta)-N \beta_{I}(\mu+\theta+\phi \sigma)\right]$ is also negative.

This completes the proof.
From the point of view of the control rate $\phi$, we can rewrite the Theorem 1 as follows.

Theorem 2. The disease-free equilibrium of the $O D E$ model (6)-(10) is locally asymptotically stable if $\phi>$ $\phi_{C}$, and unstable if $\phi<\phi_{C}$.

Next, we focus on the global stability of the ODE system (6)-10) if $R_{0}<1$. We establish the following result.

Theorem 3. The disease-free equilibrium of the $O D E$ model (6)-(10) is globally asymptotically stable whenever $R_{0}<1$.

Proof. We can integrate the equations (6)-9) to obtain

$$
\begin{aligned}
S(t)= & \int_{-\infty}^{t}\left(\mu N-\beta_{W} W S-\beta_{I} S I+\theta V\right) \\
& \times e^{-(\mu+\phi)(t-s)} \mathrm{d} s \\
V(t)= & \int_{-\infty}^{t}\left(\phi S-\sigma \beta_{I} V I\right) e^{-(\mu+\theta)(t-s)} \mathrm{d} s \\
I(t)= & \int_{-\infty}^{t}\left(\beta_{W} W S+\beta_{I} S I+\sigma \beta_{I} V I\right) \\
& \times e^{-(\gamma+\mu)(t-s)} \mathrm{d} s \\
W(t)= & \int_{-\infty}^{t} \alpha I e^{-\xi(t-s)} \mathrm{d} s .
\end{aligned}
$$

From Eqn. (18), with the substitution $x=t-s$, it follows that

$$
\limsup _{t \rightarrow \infty} W(t)=\limsup _{t \rightarrow \infty} \int_{0}^{\infty}(\alpha I(t-x)) e^{-\xi x} \mathrm{~d} x .
$$

Then we apply the following lemma introduced by Kribs-Zaleta (1999).

Lemma 1. (Kribs-Zaleta, 1999) Given a measurable sequence of non-negative uniformly bounded functions $f_{n}$,

$$
\begin{aligned}
\int \liminf f_{n} & \leq \liminf \int f_{n} \\
& \leq \limsup \int f_{n} \leq \int \limsup f_{n} .
\end{aligned}
$$

Then Eqn. (19) becomes

$$
\begin{aligned}
\limsup _{t \rightarrow \infty} W(t) & \leq \int_{0}^{\infty} \limsup _{t \rightarrow \infty} \alpha I(t-x) e^{-\xi x} \mathrm{~d} x \\
& \leq \limsup _{t \rightarrow \infty} \alpha I(t) \int_{0}^{\infty} e^{-\xi x} \mathrm{~d} x \\
& =\frac{\alpha}{\xi} \limsup _{t \rightarrow \infty} I(t) .
\end{aligned}
$$


Similarly, from Eqn. (17) we obtain

$$
\begin{aligned}
\limsup _{t \rightarrow \infty} I(t) \leq & \limsup _{t \rightarrow \infty} \int_{0}^{\infty}\left[\beta_{W} W(t-x) N\right. \\
& -V(t-x)+\beta_{I} I(t-x) N \\
& \left.-\beta_{I} V(t-x) I(t-x)(1-\sigma)\right] \mathrm{d} x \\
\leq & \limsup _{t \rightarrow \infty}\left[\beta_{w} W(t) N+\beta_{I} I(t) N\right] \\
& \times \int_{0}^{\infty} e^{-\xi x} \mathrm{~d} x \\
\leq & \frac{\left(\frac{\alpha}{\xi} N+\beta_{I} N\right)}{\gamma+\mu} \limsup _{t \rightarrow \infty} I(t) \\
= & \frac{N\left(\beta_{W} \alpha+\beta_{I} \xi\right)}{\gamma \xi+\mu \xi} \limsup _{t \rightarrow \infty} I(t) .
\end{aligned}
$$

Since $R_{0}<1$, the inequality is true only if

$$
\limsup _{t \rightarrow \infty} I(t)=0
$$

\section{Endemic equilibria}

Endemic equilibrium points are steady-state solutions where the disease persists in the population (Chitnis et al., 2008). We find that long term behavior has some important epidemiological implications such as whether an outbreak of a disease may result in an endemic situation or the infection will die out. In this section we shall conduct the endemic analysis. By setting the right-hand sides of Eqns. (6) - (10) to zero, endemic equilibria $E=$ $\left(S^{*}, V^{*}, W^{*}\right)$ are such that

$$
\begin{aligned}
S^{*} & =\frac{(\gamma+\mu)\left(\sigma \beta_{I} I^{*}+\mu+\theta\right)}{\left[\phi \sigma \beta_{I}+\left(\frac{\beta_{W} \alpha}{\xi}+\beta_{I}\right)\left(\sigma \beta_{I} I^{*}+\mu+\theta\right)\right]}, \\
V^{*} & =\frac{(\gamma+\mu) \phi}{\left[\phi \sigma \beta_{I}+\left(\frac{\beta_{W} \alpha}{\xi}+\beta_{I}\right)\left(\sigma \beta_{I} I^{*}+\mu+\theta\right)\right]}, \\
W^{*} & =\frac{\alpha I^{*}}{\xi} .
\end{aligned}
$$

In addition, we obtain a single quadratic equation for $I^{*}$,

$$
A\left(I^{*}\right)^{2}+B I^{*}+C=0
$$

where

$$
\begin{aligned}
A= & (\gamma+\mu)\left(\beta_{W} \alpha+\beta_{I} \xi\right) \sigma \beta_{I}, \\
B= & -\mu N\left(\beta_{W} \alpha+\beta_{I} \xi\right) \sigma \beta_{I}+\mu \xi(\gamma+\mu) \sigma \beta_{I} \\
& +(\gamma+\mu) \xi \phi \sigma \beta_{I}+(\gamma+\mu)\left(\beta_{W} \alpha+\beta_{I} \xi\right) \\
& \times(\mu+\theta), \\
C= & \mu\left(1-R_{C}\right) \xi(\gamma+\mu)(\mu+\theta+\phi) .
\end{aligned}
$$

Note that $A=0$ if and only if $\sigma=0$. When $A=0$, the endemic equilibrium is unique and exists only if $R_{C}>$ 1. In what follows, we must assume that $0<\sigma<1$ (consistent with the assumption in Section 2). If $\sigma=0$, $A=0$, then $A\left(I^{*}\right)^{2}+B I^{*}+C=0$ is a linear equation with a unique solution

$$
I^{*^{\prime}}=\frac{(\gamma+\mu) R_{C}^{\prime}}{\mu N\left(R_{C}^{\prime}-1\right)},
$$

where

$$
R_{C}^{\prime}=\frac{\beta_{W} \alpha N(\mu+\theta)+\beta_{I} \xi N(\mu+\theta)}{\xi(\gamma+\mu)(\mu+\theta+\phi)} .
$$

Clearly, the sign of $C$ depends on the value of $R_{C}$. It is obvious that $A>0$ since all parameters are positive. The two non-zero roots of Eqn. (22), $I_{1}$ and $I_{2}$, must satisfy

$$
I_{1} I_{2}=\frac{C}{A}, \quad I_{1}+I_{2}=-\frac{B}{A} .
$$

When $R_{C}>1, C<0, B^{2}-4 A C>0$, there is one and only one positive real root. If $R_{C}<1$, then $C>0$, which yields

$$
N\left(\beta_{W} \alpha+\beta_{I} \xi\right)<\xi(\gamma+\mu) .
$$

Thus

$$
\begin{aligned}
B= & \mu \sigma \beta_{I}\left[\xi(\gamma+\mu)-N\left(\beta_{W} \alpha+\beta_{I} \xi\right)\right] \\
& +(\gamma+\mu) \xi \phi \sigma \beta_{I}+(\gamma+\mu)\left(\beta_{W} \alpha+\beta_{I} \xi\right) \\
> & 0 .
\end{aligned}
$$

Hence, here we have either two negative real roots, or two complex conjugate roots with negative real parts of Eqn. (22). There is no positive endemic equilibrium. by
The Jacobian matrix for the system (6)-(10) is given 


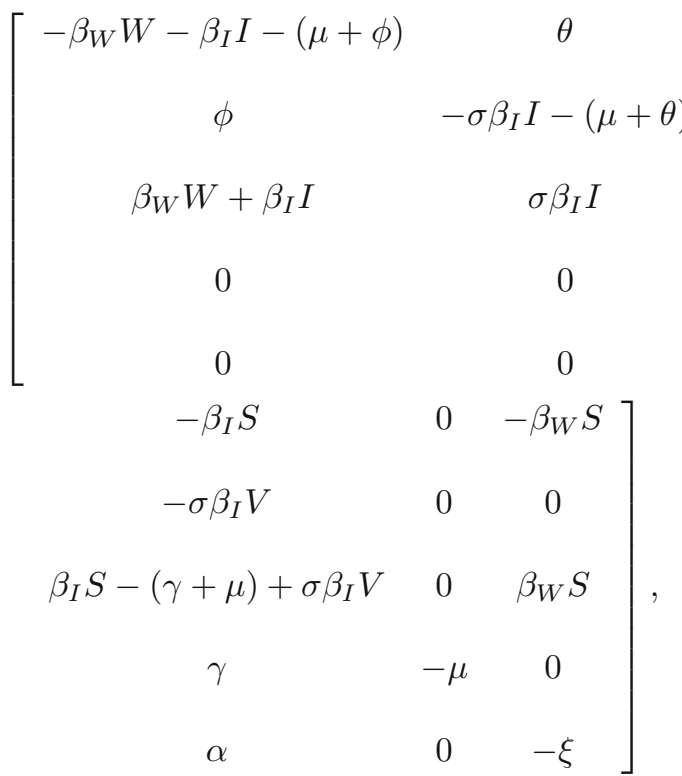

where the characteristic polynomial of the matrix $J_{B}^{*}$ is found as

$$
\begin{aligned}
& \operatorname{det}\left(\lambda I-J_{B}^{*}\right) \\
& \quad=(\lambda+\mu)\left[a_{4} \lambda^{4}+a_{3} \lambda^{3}+a_{2} \lambda^{2}+a_{1} \lambda+a_{0}\right],
\end{aligned}
$$

whose coefficients $a_{i}$ in terms of the elements of $J_{B}^{*}$ are

$$
\begin{aligned}
a_{4}= & 1, \\
a_{3}= & \beta_{W} W^{*}+\beta_{I} I^{*}+\sigma \beta_{I} I^{*}+2 \mu+\phi+\theta+\xi \\
& +\left(\gamma+\mu-\beta_{I} S^{*}-\sigma \beta_{I} V^{*}\right), \\
a_{2}= & \xi\left(\sigma \beta_{I} I^{*}+\mu+\theta\right)+\xi\left(\beta_{W} W^{*}+\beta_{I} I^{*}+\mu+\phi\right) \\
& +\left(-\beta_{I} S^{*}-\sigma \beta_{I} V^{*}+\gamma+\mu\right)\left(\sigma \beta_{I} I^{*}+\mu+\theta\right) \\
& +\left(-\beta_{I} S^{*}-\sigma \beta_{I} V^{*}+\gamma+\mu\right) \\
& \times\left(\beta_{W} W^{*}+\beta_{I} I^{*}+\mu+\phi\right) \\
& +\left(\beta_{W} W^{*}+\beta_{I} I^{*}+\mu\right)\left(\sigma \beta_{I} I^{*}+\mu+\theta\right) \\
& +\phi\left(\sigma \beta_{I} I^{*}+\mu\right) \\
& +\sigma^{2} \beta_{I}^{2} V^{*} I^{*}+\beta_{W} W^{*}+\beta_{I} I^{*}, \\
a_{1} & \xi\left(\beta_{W} W^{*}+\beta_{I} I^{*}+\mu\right)\left(\sigma \beta_{I} I^{*}+\mu+\theta\right) \\
& +\left[\left(\beta_{W} W^{*}+\beta_{I} I^{*}+\mu\right)\left(\sigma \beta_{I} I^{*}+\mu+\theta\right)\right. \\
& \left.+\phi\left(\sigma \beta_{I} I^{*}+\mu\right)\right] \\
& \times\left(-\beta_{I} S^{*}-\sigma \beta_{I} V^{*}+\gamma+\mu\right)+\xi \phi\left(\sigma \beta_{I} I^{*}+\mu\right) \\
& +\left(\sigma \beta_{I} I^{*}+\mu+\theta+\xi+\alpha\right)\left(\beta_{W} W^{*}+\beta_{I} I^{*}\right) \\
& +\left(\beta_{W} W^{*}+\beta_{I} I^{*}+\mu+\phi+\xi\right) \sigma^{2} \beta_{I}^{2} V^{*} I^{*} \\
& +\phi^{2} \beta_{I}^{2} S^{*} I^{*}, \\
= & \left(\beta_{W} W^{*}+\beta_{I} I^{*}+\mu+\phi\right) \xi \sigma^{2} \beta_{I}^{2} V^{*} I^{*} \\
& +\theta \sigma \beta_{I} V^{*}\left(\beta_{W} W^{*}+\beta_{I} I^{*}\right)+\xi \phi^{2} \beta_{I}^{2} S^{*} I^{*} \\
& +(\xi+\alpha)\left(\sigma \beta_{I} I^{*}+\mu+\theta\right)\left(\beta_{W} W^{*}+\beta_{I} I^{*}\right) \\
& +\xi \phi\left(\sigma \beta_{I} I^{*}+\mu\right)\left(-\beta_{I} S^{*}-\sigma \beta_{I} V^{*}+\gamma+\mu\right) .
\end{aligned}
$$

The criterion that provides necessary and sufficient conditions for the endemic equilibrium to be asymptotically stable is the Routh-Hurwitz criterion (Korn et al., 2000), which requires

$$
\begin{gathered}
a_{3}>0, \quad a_{1}>0, \quad a_{0}>0, \\
a_{1}\left(a_{2} a_{3}-a_{1}\right)>a_{0} a_{3}^{2} .
\end{gathered}
$$

From Eqn. [8], it is easy to see that

$$
\gamma+\mu-\beta_{I} S-\sigma \beta_{I} V>0 .
$$

Thus $a_{3}>0, a_{1}>0$ and $a_{0}>0$.

To prove the inequality $a_{1}\left(a_{2} a_{3}-a_{1}\right)>a_{0} a_{3}^{2}$, it is convenient to prove the following two inequalities:

$$
\begin{aligned}
& a_{1} a_{2} a_{3}>2 a_{1}^{2}, \\
& a_{1} a_{2} a_{3}>2 a_{0} a_{3}^{2} .
\end{aligned}
$$

These inequalities can be verified by direct calculation (details omitted), establishing the following theorem.

Theorem 4. The positive endemic equilibrium of the ODE system (6)-(10) is locally asymptotically stable if $R_{C}>1$.

In epidemiology, bifurcation theory is concerned with how solutions of a differential equation depend on threshold parameters, and it can explain how the changes in dynamics take place from a resting state to oscillations. It plays a relevant role in disease control and eradication. Next, we show that there are no backward bifurcations possible when $R_{C}<1$ and $B>0$. From $R_{C}<1$ it follows that

$$
\begin{aligned}
\beta_{W} \alpha N(\mu+\theta) & +\beta_{I} \xi N(\mu+\theta+\sigma \phi) \\
& <\xi(\gamma+\mu)(\mu+\theta+\phi),
\end{aligned}
$$

that is,

$$
N(\mu+\theta)\left(\beta_{W} \alpha+\beta_{I} \xi\right)<\xi(\gamma+\mu)(\mu+\theta+\phi) .
$$

Substituting this inequality into the expression 25 of $B$, the first term of $B$ satisfies

$$
\begin{aligned}
\mu N\left(\beta_{W} \alpha+\beta_{I} \xi\right) \sigma \beta_{I} & <\frac{\mu \sigma \beta_{I} \xi(\gamma+\mu)(\mu+\theta+\phi)}{\mu+\theta} \\
& <\mu \sigma \beta_{I} \xi(\gamma+\mu) .
\end{aligned}
$$

The other terms of $B$ are all positive. Thus $B>0$ whenever $R_{C}<1$. Hence, we have a forward bifurcation when $R_{C}=1$. This result contrasts with the well-known analysis of some regular SIR epidemic models (Brauer, 2000; Van den Driessche et al., 2000; Kribs-Zaleta and Mertcherab, 2002; Arino et al., 2003; Chitnis et al., 2006). 
From this conclusion, when $R_{C}<1$, the infectious number decreases to zero. When $R_{C}>1$, the infectious number either increases or decreases to the curve that represents the endemic equilibrium which is stable. We can predict that significant changes make oscillations during the long term period.

Theorem 5. The ODE model system (6)-(10) has a forward bifurcation at $R_{C}=1$.

\section{Simulation results}

4.1. Vaccination free model. Cholera is one of the worst waterborne diseases and is not easy to control. In order to better understand the serious cholera outbreak as well as for possible prediction and control of future cholera epidemics, we apply the vaccination free model (11)-(4) and the vaccination model (6)-(10) to study the cholera dynamics. As an example, we use the data of the 2008-2009 cholera outbreak in Zimbabwe to verify the models. The Zimbabwe cholera outbreak began in August 2008, swept to all of Zimbabwe's ten provinces, and spread to Botswana, Mozambique, South Africa and Zambia quickly. The case fatality rate for the outbreak was higher than expected for such outbreaks. On 4 December 2008, the Zimbabwe government declared the outbreak to be a national emergency and requested international aid (WHO). The principal cause of the outbreak is the lack of safe water in urban areas and communities. Many places had no water supply for much longer during the outbreak period. By March 2009 the World Health Organization estimated that 4,011 people had succumbed to the waterborne disease and 91,164 cases were infected.

Since $\beta_{I}$ and $\beta_{W}$ vary from place to place, in our Zimbabwe case we need to adjust these two parameters to match the reported infections data published by the WHO for the period from the end of August 2008 to March, 2009. Since the total population in Zimbabwe is $12,347,240$, in order to make the calculation simpler we scale down all data numbers by a factor of 1,200. All epidemiological parameter values for cholera in the literature are given in Table 1 . When $\beta_{W}=0.00000002$, $\beta_{I}=0.0001568$, the model prediction can well fit the

Table 1. Model parameters and values.

\begin{tabular}{|c|c|c|}
\hline Parameter & Symbol & Value \\
\hline \hline Natural human birth/death rate & $\mu$ & $(35 y)^{-1}$ \\
\hline Shedding rate & $\alpha$ & 10 cells/ml.day \\
\hline Bacterial death rate & $\xi$ & $(30 d)^{-1}$ \\
\hline Recovery rate & $\gamma$ & $(5 d)^{-1}$ \\
\hline Vaccination rate & $\phi$ & Variable \\
\hline Waning rate & $\theta$ & Variable \\
\hline Vaccine efficacy rate & $\sigma$ & Variable \\
\hline
\end{tabular}

data. After substituting all parameters into Eqn. (5), $R_{0}=1.163$, showing cholera would spread in Zimbabwe. Figure 1 shows the data fitting for the infected population $I$, where the curve represents the model prediction and the squares mark the reported Zimbabwean data.

To verify the model prediction, we run the numerical simulation for a long time interval. Figures 2 and 3 show the results for $I, S$ and $R$ of the vaccination free model with the initial condition $I(0)=1, S(0)=9999, R(0)=$ $B(0)=0$. By substituting all parameter values into Eqns. (1)-(4), one can obtain the unique positive endemic equilibrium $\left(I^{*}, S^{*}, R^{*}\right)=(0.442,8699.5,1300)$. In Fig. 2. cholera outbreak reaches the first peak at $t=22.5$ weeks with the value 76 (normalized value), then drops to almost zero, which means the disease is gradually eradicated from the population. However, next a smaller peak appears with a smaller value for $I$ as 23 at $t=325$ weeks, and several oscillations later, showing cholera will restart again and again until $I$ reaches a steady-state value 0.442 , and after that no more outbreaks are triggered. Figure 3 shows almost the same trend with different magnitude, susceptible population $S$ and recovered population $R$ appear the first pulse at $t=$ 22.5 weeks with peak value around 7,700, and 2,224, respectively. Finally, $S$ and $R$ reach their steady-state value 8699.5 and 1300 , respectively.

4.2. Vaccination model. For the vaccination model (6)-(10), since the control reproduction number $R_{C}$, which provides useful guidelines for the prevention and

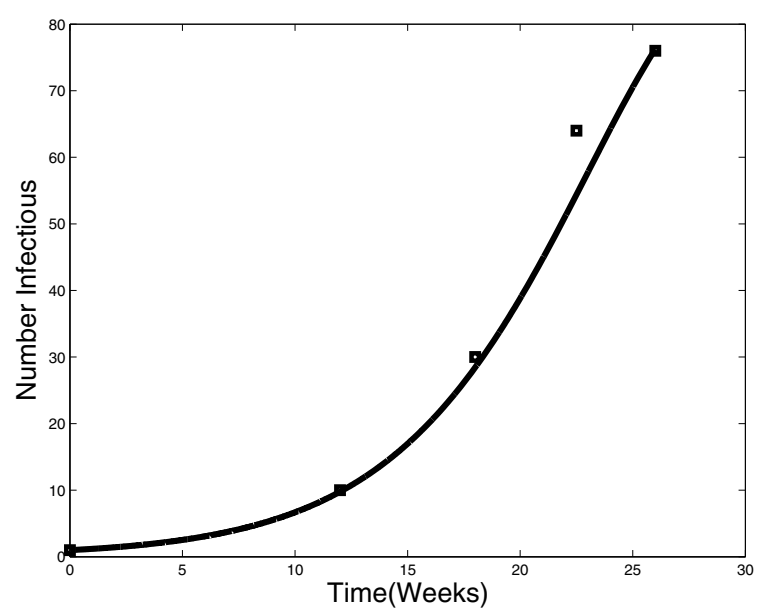

Fig. 1. Figure of $I$ vs. time (weeks) shows data fitting for the cholera outbreak in Zimbabwe, where the curve represents the model prediction and the squares mark the reported data. After normalized by a factor of 1,200 , the cholera outbreak had 10, 30, 66 and 76 (normalized) cholera infections after 12 weeks, 18 weeks, 22.5 weeks and 26.5 weeks, respectively, from the beginning of the outbreak. 
control strategies on cholera epidemics in Zimbabwe, strongly depends on the parameters $\beta_{W}, \beta_{I}, \phi, \sigma$, and $\theta$, in order to determine which of the parameters are most influential on model output, we use the Latin hypercube sampling approach (Blower and Dowlatabadi, 1994; Marino et al., 2008) to incorporate the uncertainty in such five parameters into the model. A sensitive analysis can be performed by calculating Partial Rank Correlation Coefficients (PRCCs) for each parameter. The magnitude of the PRCC indicates the importance of the uncertainty in contributing to the imprecision in predicting the value of the parameters. As shown in Table 2, we pick the total infection $I$ as the outcome and use sample sizes $n=1000$ and $n=2000$, respectively. Here $\beta_{I}$ and $\beta_{W}$ are more sensitive than other parameters. Especially, $\beta_{I}$ is

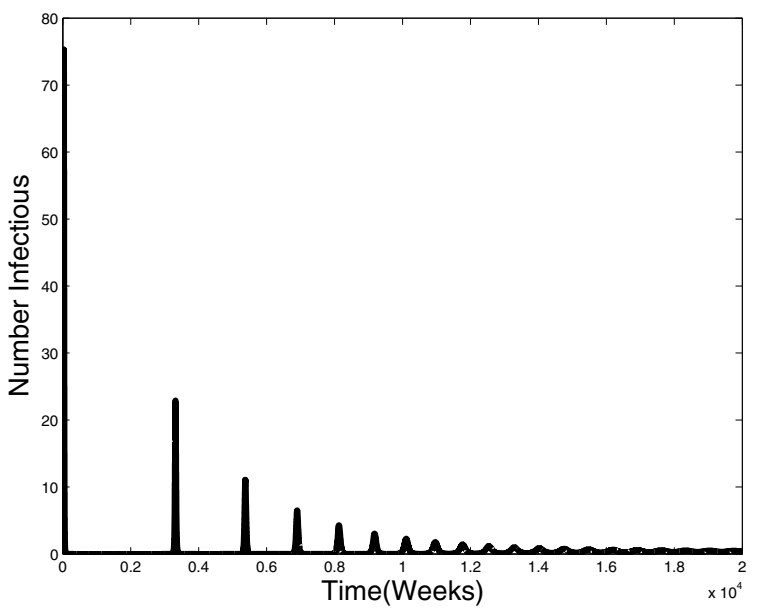

Fig. 2. Result for $I$ vs. time (weeks) of a vaccination free model with the initial condition $I(0)=1, S(0)=9999$, $R(0)=B(0)=0$.

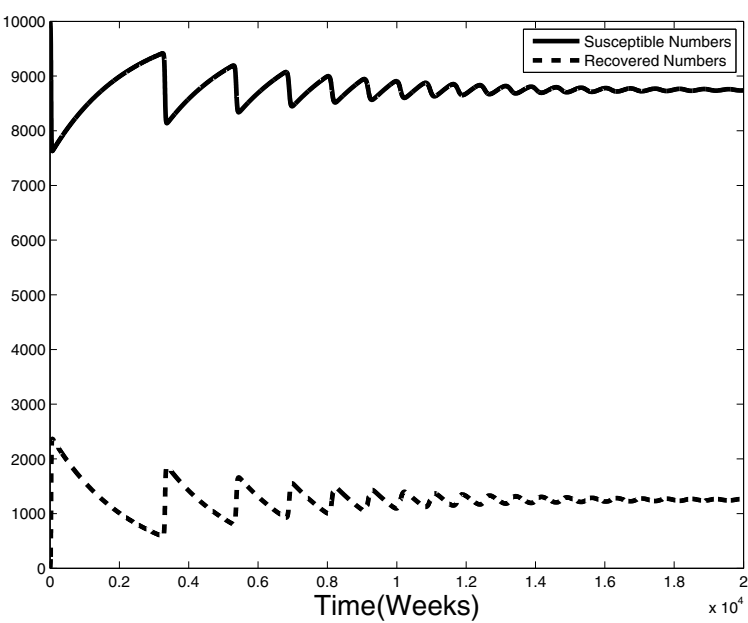

Fig. 3. Result for $S, R$ vs. time (weeks) of a vaccination free model with the initial condition $I(0)=1, S(0)=9999$, $R(0)=B(0)=0$. the most influential parameter in determining $R_{C}$.

If we rewrite the expression for $R_{C}$ as

$$
R_{C}=1+\frac{E I^{2}+F I}{D I+H}
$$

where

$$
\begin{aligned}
E= & (\gamma+\mu)\left(\beta_{W} \alpha+\beta_{I} \xi\right) \sigma \beta_{I}, \\
F= & {\left[\mu \sigma^{2} \beta_{I}^{2} N^{2} \xi \phi-\mu N \sigma \beta_{I} \xi(\gamma+\mu) \phi\right.} \\
& \left.+(\gamma+\mu)^{2}(\mu+\theta) \xi(\mu+\theta+\phi)\right] / N(\mu+\theta), \\
H= & \mu \xi(\gamma+\mu)(\mu+\theta+\phi),
\end{aligned}
$$

then Eqn. 277 represents approximately a curve passing the bifurcation point $R_{C}=1$. After substituting all parameters into Eqn. (27) and setting $\phi=0.5, \sigma=0.2$, $\theta=0.5$, a bifurcation diagram is illustrated in Fig. 4 providing a picture of how these equilibria depend on $R_{C}$ and how stability exchanges at point $R_{C}=1$.

Take the economic reason into consideration. Vaccination strategies cost a lot, so if we want to succeed in vaccinating the disease and bringing the control reproduction number less than one, it is necessary to find the minimum control reproduction number $R_{C}$, that is, a certain vaccination rate $\phi^{*}$ can reduce $R_{C}$ to a value which is less than one as $\sigma$ and $\theta$ are fixed. Thus

$$
\phi^{*}>\frac{(\mu+\theta)\left[\beta_{W} \alpha N+\beta_{I} \xi N-\xi(\gamma+\mu)\right]}{\xi\left[(\gamma+\mu)-\beta_{I} N \sigma\right]} .
$$

Table 2. Sensitivity analysis.

\begin{tabular}{|c|c|c||c|c|c|}
\hline & $\beta_{w}$ & $\beta_{I}$ & $\phi$ & $\sigma$ & $\theta$ \\
\hline \hline Prcc Value $(n=1000)$ & 0.6298 & 0.8885 & 0.3284 & 0.0558 & 0.03646 \\
\hline Prcc Value $(n=2000)$ & 0.5878 & 0.8912 & 0.2109 & 0.08665 & 0.07032 \\
\hline
\end{tabular}

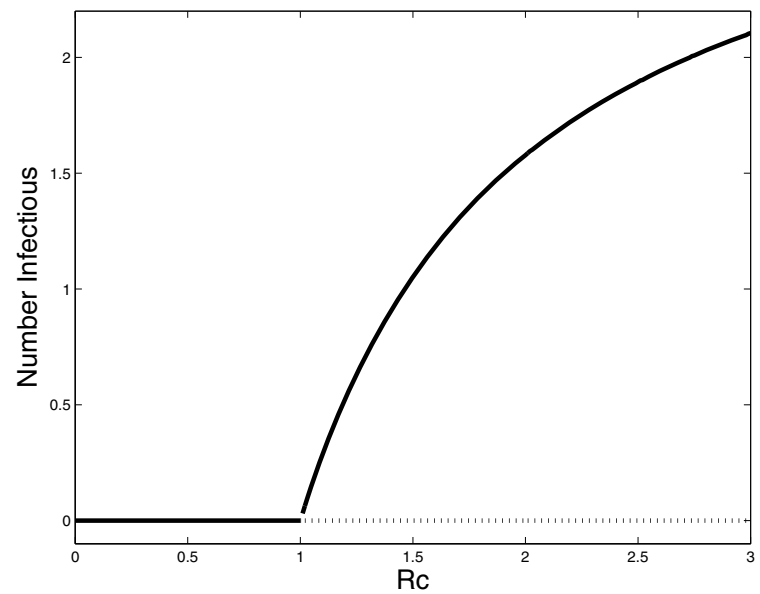

Fig. 4. Plot of $I$ vs. $R_{C}$ shows a forward bifurcation with $\phi=$ $0.5, \sigma=0.2, \theta=0.5$, other parameters being given in Table 1. The solid lines represent the stable equilibria, and the dashed line represents the unstable equilibrium. There is a stability exchange at $R_{C}=1$. 
Here, we consider the parameter values $\sigma=0.2$ (vaccine is $80 \%$ effective), $\theta=0.5$, based on (28). We need $\phi>$ 0.10 , hence $R_{C}<1$. As shown in Fig. 5, we set the initial condition as $I(0)=100, V(0)=100, S(0)=9800$, $R(0)=B(0)=0$, and choose parameter values $\phi=0.3$, $\sigma=0.2$, and $\theta=0.5$. In this case, $R_{C}=0.81$ and the disease quickly dies out $(I=0)$, so that it can be eradicated. It is important to note that since the collapse of Zimbabwe's public health system, hospitals lacked basic medicines and staff, and this vaccination standard was not met in Zimbabwe.

Similarly, we now present numerical simulations in order to verify the vaccination model prediction with the initial condition $I(0)=1, V(0)=1, S(0)=9998$, $R(0)=B(0)=0$, and the parameter values $\phi=0.05$,

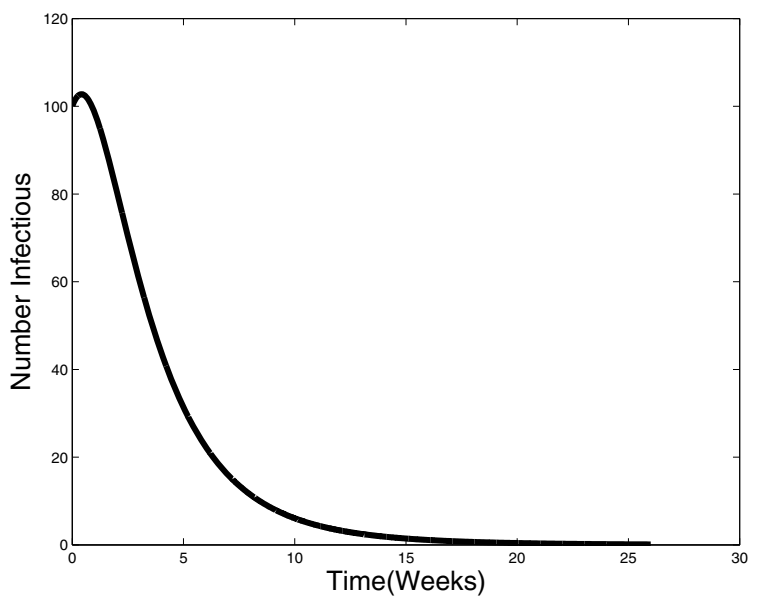

Fig. 5. Result for $I$ vs. time (weeks) with the initial condition $I(0)=100, V(0)=100, S(0)=9800, R(0)=$ $B(0)=0$, parameter values $\phi=0.3, \sigma=0.2$, and $\theta=0.5$.

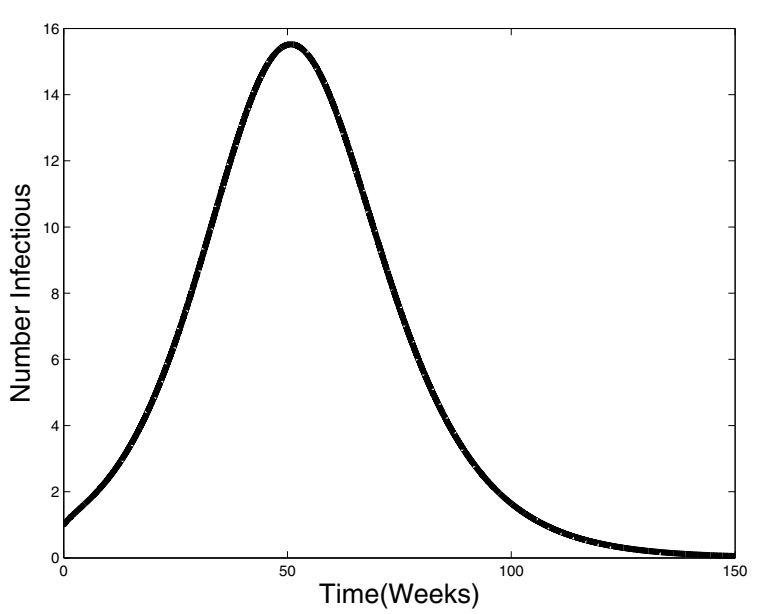

Fig. 6. Result for $I$ vs. time (weeks) with the initial condition $I(0)=1, V(0)=1, S(0)=9998, R(0)=B(0)=0$, parameter values $\phi=0.05, \sigma=0.2$, and $\theta=0.5$. $\sigma=0.2, \theta=0.5$. Under this setting, $R_{C}=1.08$. Figure 6 shows the cholera outbreak will be trigged with a peak value around 15, which is much lower than that of the vaccination free model, showing the vaccination strategies do weaken the cholera outbreak even though they do not eradicate the outbreak. If we consider a much more longer period, a single positive endemic equilibrium exists, $\left(I^{*}, V^{*}, S^{*}, R^{*}\right)=(0.2263,843.2,8439.8,716.78)$. As shown in Figs. 7 and 8 , after the first outbreak with a lower magnitude, there exist several oscillations until $I$ drops to 0.2263 . $V, S$ and $R$ reach their steady-state values at $843.2,8439.8,716.78$, respectively. Thus, our vaccination model predicts that, even with the vaccination, and if $\phi<\phi^{*}$, there will be a cholera outbreak every 70 years in Zimbabwe, and that the disease will remain endemic in the long term finally.

\section{Conclusion}

We presented a vaccination model (6)-(10) for the transmission of cholera, with multiple transmission pathways: direct human-to-human and indirect water-to-human modes, which differentiate cholera from many other infectious diseases. The basic reproduction number as modified by vaccination, namely, control reproduction number $R_{C}$ is derived by (12) using the next generation matrix method. It is a key parameter in our model. We showed that if $R_{C}$ is less than one, the disease dies out and the disease-free equilibrium is stable. If $R_{C}$ is greater than one, the disease persists and the disease free equilibrium is unstable while the positive endemic equilibrium is stable. The forward bifurcation occurs at the critical point $R_{C}=1$ (see Fig. 4).

We have to note that it is generally difficult to show the global stability for the endemic equilibrium

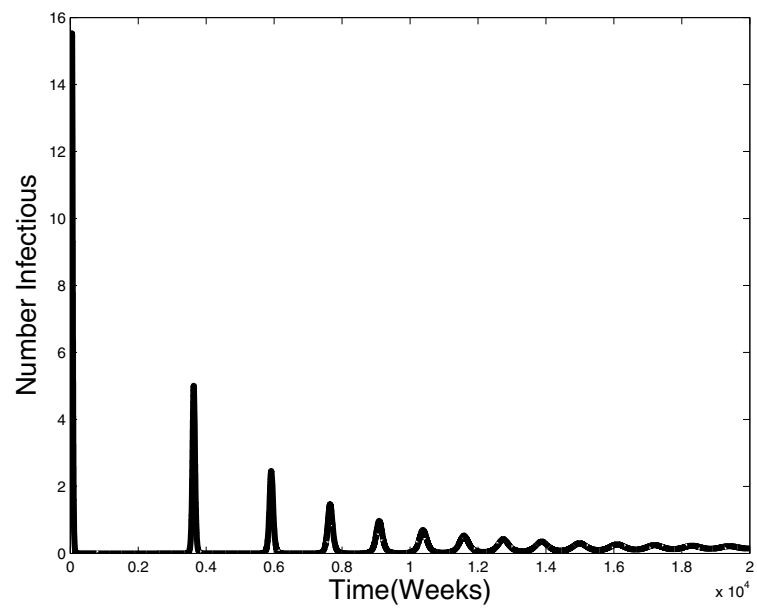

Fig. 7. Infected population $I$ vs. time (weeks) of a vaccination model with the initial condition $I(0)=1, V(0)=1$, $S(0)=9998, R(0)=B(0)=0$. 
for high-dimensional nonlinear systems, such as our vaccination model (6)-10, no matter which methods we use-the classic Lyapunov functions or the classical Poincaré-Bendixson framework, the theory of monotone flows-Lipschitz manifolds, etc. (Moghadas and Gumel, 2002; Zhang and Ma, 2003; Li and Zhen, 2005). Recently, a few efforts (Feckan, 2001; Sanchez, 2010) provide useful directions for our future work to study the global stability for high-dimensional models. We only present the graph trajectory of $S$ vs. $I$ in Fig. 9 with three different initial conditions, and one can see that the endemic equilibrium seems to be globally stable if $R_{C}>1$.

We must point out that vaccination is not the only control measure against a cholera outbreak. Water sanitation, medical therapy and antibiotic treatment are possible prevention and intervention strategies. Also, the vaccination does not always work out due to the limitations of the medical development level and financial budget, which is also a restriction in our study. However, it is not possible to extend this vaccination model to other kinds of diseases, such as HIV, since there is no vaccine for HIV yet (Gani et al., 1997). Moreover, in this paper, we consider the vaccination as a continuous state, since sometimes the vaccination process is discontinuous or seasonal, it can be modeled by impulsive differential equations, which is one of our future works.

\section{Acknowledgment}

This work was partially supported by the NSF of China (No. 11226261, Tianyuan Fund for Mathematics), (No. 11271388), the Natural Science Foundation of CQ (No. CSTC 2011BB0104, No. CSTC 2011BB0105) and the Research Foundation of the Chongqing Municipal

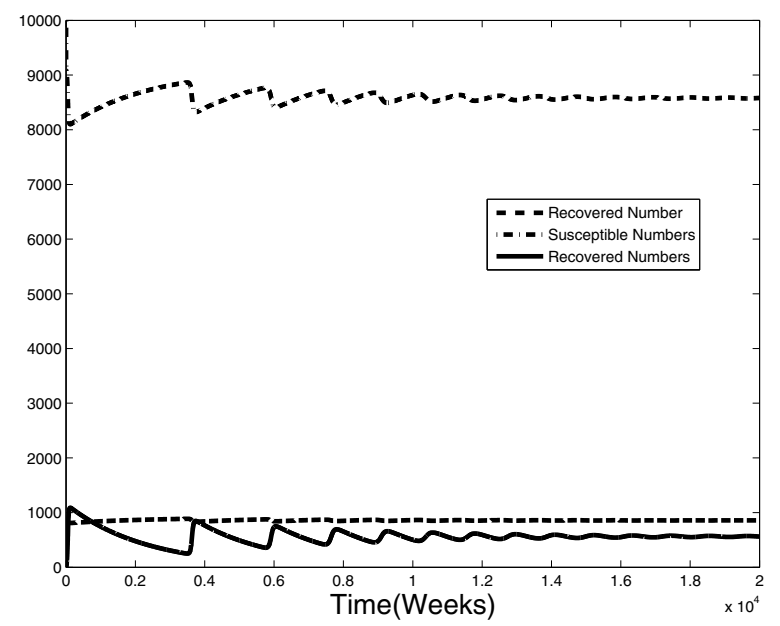

Fig. 8. Result for $S, R, V$ vs. time (weeks) of a vaccination model with the initial condition $I(0)=1, V(0)=1$, $S(0)=9998, R(0)=B(0)=0$.

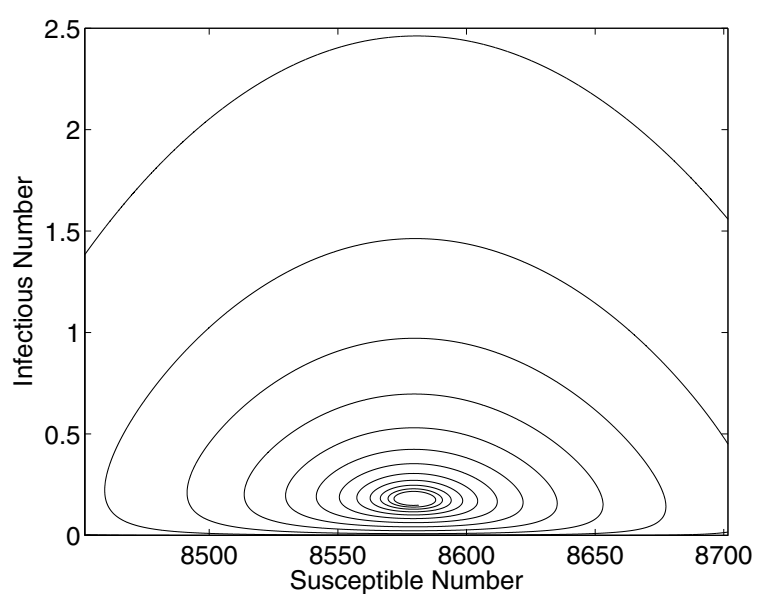

(a)

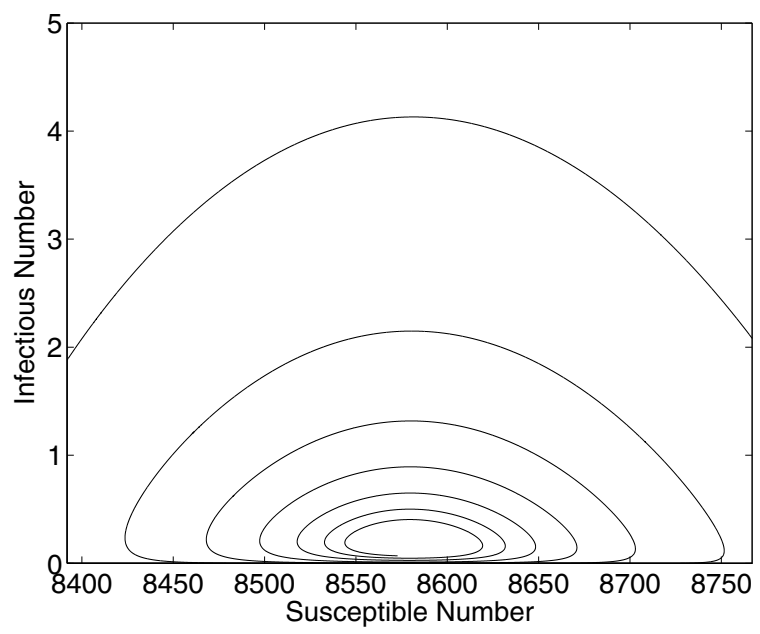

(b)

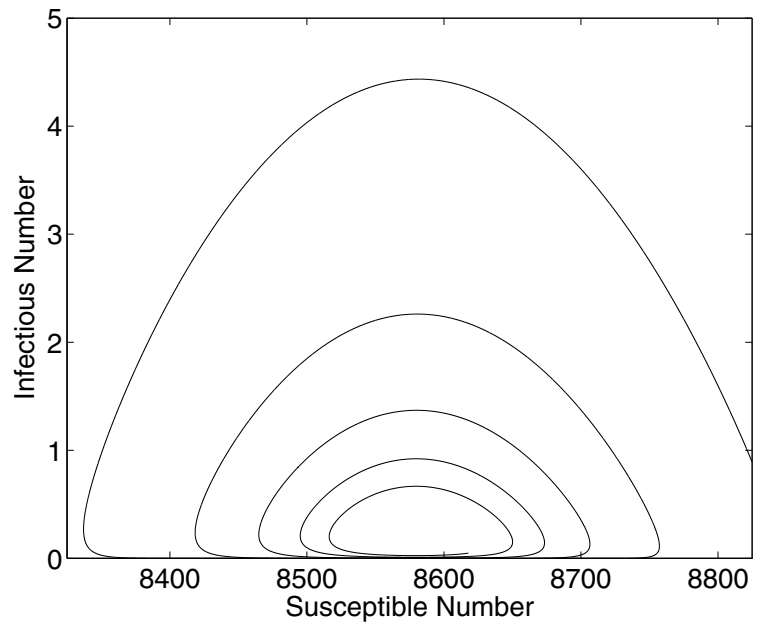

(c)

Fig. 9. Endemic equilibrium point of $I$ vs. $S$ with three different initial conditions: $I_{1}=1$ (a), $I_{2}=100$ (b), $I_{3}=200$ (c). 
Education Commission (No. KJ130719, No. KJ130730).

\section{References}

Anderson, R.M. and May, R.M.(1990). Infectious Diseases of Humans: Dynamics and Control, Oxford University Press, Oxford.

Arino, J.C., McCluskey, C. and van den Driessche P. (2003). Global results for an epidemic model with vaccination that exhibits backward bifurcation, SIAM Journal on Applied Mathematics, 64(1): 260-276.

Blayneh, K.W., Gumel, A.B., Lenhart, S. and Clayton, T. (2010). Backward bifurcation and optimal control in transmission dynamics of West Nile Virus, Bulletin of Mathematical Biology 72(4): 1006-1028.

Brauer, F. (2004). Backward bifurcations in simple vaccination models, Journal of Mathematical Analysis and Application 298(2): 418-431.

Blower, S.M. and Dowlatabadi, H. (1994). Sensitivity and uncertainty analysis of complex models of disease transmission: An HIV model, as an example, International Statistical Review 62(2): 229-243.

Buonomo, B. and Lacitignola, D. (2011). On the backward bifurcation of a vaccination model with nonlinear incidence, Nonlinear Analysis: Modelling and Control 16(1): 30-46.

Buonomo, B. and Lacitignola, D. (2008). On the dynamics of an SEIR epidemic model with a convex incidence rate, Ricerche di Matematica 57(2): 261-281.

Buonomo, B. and Lacitignola, D. (2010). Analysis of a tuberculosis model with a case study in Uganda, Journal of Biological Dynamics 4(6): 571-593.

Castillo-Chavez, C. and Song, B.(2004). Dynamical models of tubercolosis and their applications, Mathematical Biosciences and Engineering 1(2): 361-404.

Capasso, V. and Paveri-Fontana, S.L. (1979). A mathematical model for the 1973 cholera epidemic in the European Mediterranean region, Revue D'Epidémiologie de Santé Publique 27(2): 121-132.

Chitnis, N., Cushing, J.M. and Hyman, J.M.(2006). Bifurcation analysis of a mathematical model for malaria transmission, SIAM Journal of Applied Mathematics 67(1): 24-45.

Chitnis, N., Cushing, J.M. and Cushing, J.M. (2008). Determining important parameters in the spread of malaria through the sensitivity analysis of a mathematical model, Bulletin of Mathematical Biology 79(5): 1272-1296.

Codeço, C.T. (2001). Endemic and epidemic dynamics of cholera: The role of the aquatic reservoir, BMC Infectious Diseases, 1:1

Dietz, K. and Schenzle, D.(1985). Mathematical models for infectious disease statistics, in A.C. Atkinson and S.E. Fienberg (Eds.), Centenary Volume of the International Statistical Institute, Springer-Verlag, Berlin, pp. 167-204.

Feckan, M. (2001). Criteria on the nonexistence of invariant Lipschitz submanifolds for dynamical systems, Journal of Differential Equations 174(2): 392-419.
Gani, J., Yakowitz, S. and Blount, M. (1997). The spread and quarantine of HIV infection in a prison system, SIAM Journal on Applied Mathematics 57(6): 1510-1530.

Gumel, A.B. and Moghadas, S.M. (2003). A qualitative study of a vaccination model with non-linear incidence, Applied Mathematics and Computation 143(2-3): 409-419.

Hartley, D.M., Morris, J.G. and Smith, D.L. (2006). Hyperinfectivity: A critical element in the ability of $V$. cholerae to cause epidemics?, PLoS Medicine 3(1): 63-69.

Hethcote, H.W. (2000). The mathematics of infectious diseases, SIAM Review 42(4):599-653.

Korn, G.A. and Korn, T.M. (2000). Mathematical Handbook for Scientists and Engineers: Definitions, Theorems, and Formulas for References and Review, Dover Publications, Mineola, NY.

Kribs-Zaleta, C.M. (1999). Structured models for heterosexual disease transmission, Mathematical Biosciences 160(1): 83-108.

Kribs-Zaleta, C.M. and Martchevab, M. (2002). Vaccination strategies and backward bifurcation in an age-since-infection structured model, Mathematical Biosciences 177/178: 317-332.

Kribs-Zaleta, C.M. and Velasco-Hernandez, J.X. (2000). A simple vaccination model with multiple endemic states, Mathematical Biosciences 164(2): 183-201.

Li, G. and Zhen, J. (2005). Global stability of an SEI epidemic model with general contact rate, Chaos Solitons \& Fractals 23(3): 997-1004.

Liao, S. and Wang, J. (2011). Stability analysis and application of a mathematical cholera model, Mathematical Biosciences and Engineering 8(3):733-752.

Liu, X. and Wang, C. (2010). Bifurcation of a predator-prey model with disease in the prey, Nonlinear Dynamics 62(4):841-850.

Marino, S., Hogue, I., Ray, C.J. and Kirschner, D.E.(2008). A methodology for performing global uncertainty and sensitivity analysis in system biology, Journal of Theoretical Biology 254(1): 178-196.

Moghadas, S.M. and Gumel, A.B. (2002). Global stability of a two-stage epidemic model with generalized non-linear incidence, Mathematics and Computers in Simulation 60(1-2): 107-118.

Mukandavire, Z., Liao, S., Wang, J., Gaff, H., Smith, D.L. and Morris, J.G. (2011). Estimating the reproductive numbers for the 2008-2009 cholera outbreaks in Zimbabwe, Proceedings of the National Academy of Sciences of the United States of America 108(21): 8767-8772.

Samsuzzoha, M.D., Singh, M. and Lucy, D. (2012). A numerical study on an influenza epidemic model with vaccination and diffusion, Applied Mathematics and Computation 219(1): 122-141.

Song, X., Jiang, Y. and Wei, H.(2009). Analysis of a saturation incidence SVEIRS epidemic model with pulse and two time delays, Applied Mathematics and Computation 214(2): 381-390. 
Szymańska, Z. (2013). Analysis of immunotherapy models in the context of cancer dynamics, International Journal of Applied Mathematics and Computer Science 13(3): 407-418.

Sanchez, L.A. (2010). Existence of periodic orbits for high-dimensional autonomous systems, Journal of Mathematical Analysis and Applications 363(2): 409-418.

Tien, J.H. and Earn, D.J.D. (2010). Multiple transmission pathways and disease dynamics in a waterborne pathogen model, Bulletin of Mathematical Biology 72(6): 1502-1533.

Van den Driessche, P. and Watmough, J.(2002). Reproduction numbers and sub-threshold endemic equilibria for compartmental models of disease transmission, Mathematical Biosciences 180(1-2): 29-48.

Van den Driessche, P. and Watmough, J. (2000). A simple SIS epidemic model with a backward bifurcation, Journal of Mathematical Biology 40(6): 525-540.

Vynnycky, E., Trindall, A. and Mangtani, P.(2007). Estimates of the reproduction numbers of Spanish influenza using morbidity data, International Journal of Epidemiology 36(4): 881-889.

Yildirim, A. and Cherruault, Y.(2009). Analytical approximate solution of a SIR epidemic model with constant vaccination strategy by homotopy perturbation method, Kybernetes 38(9): 1566-1575.

Yu, H.G., Zhong, S.M., Agarwal, R.P. and Xiong L.L. (2010). Species permanence and dynamical behaviour analysis of an impulsively controlled ecological system with distributed time delay, Computers and Mathematics with Applications 59(2): 3824-3835.
Zhang, X. and Liu, X. (2009). Backward bifurcation and global dynamics of an SIS epidemic model with general incidence rate and treatment, Nonlinear Analysis: Real World Applications 10(2): 565-575.

Zhang, J. and Ma, Z. (2003). Global dynamics of an SEIR epidemic model with saturating contact rate, Mathematical Biosciences 185(1): 15-32.

World Health Organization (2010). Zimbabwe, http://www.who.int/countries/zwe/en/

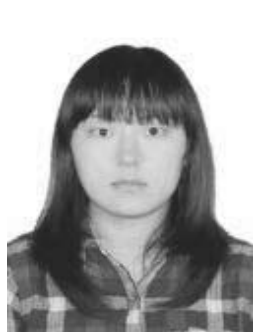

Shu Liao received her Ph.D. in 2010 from Old Dominion University, USA. Currently she is an assistant professor at the School of Mathematics and Statistics, Chongqing Technology and Business University in China. Her main research interests are mathematical biology, dynamical systems and fluid dynamics.

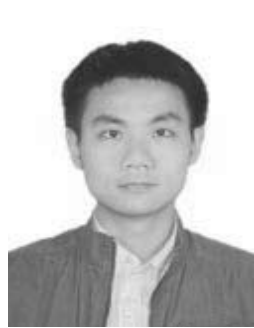

Weiming Yang is an assistant professor at the School of Mathematics and Statistics, Chongqing Technology and Business University in China. He got his first master degree in applied mathematics from the University of Toledo, and his second master degree and Ph.D. in statistics from Old Dominion University. His research area involves longitudinal data analysis, time series and copula models.

Received: 23 January 2013

Revised: 16 June 2013

Re-revised: 31 July 2013 